\title{
Salinomicina e semduramicina associadas em diferentes concentraçóes no controle da eimeriose em frangos de corte
}

Salinomycin and semduramicin in different concentrations on the broilers eimeriosis control

Giankleber Strumielo Diniz ${ }^{1 *}$; Anderlise Borsoi²; Juarez Morbini Lopes ${ }^{3}$; João Luis Garcia; José da Silva Guimarães Junior ${ }^{1}$

\author{
${ }^{1}$ Universidade Estadual de Londrina - UEL \\ ${ }^{2}$ Universidade Federal do Rio Grande do Sul - UFRGS \\ ${ }^{3}$ Universidade Federal de Santa Maria - UFSM \\ Recebido em 12 de Dezembro de 2008 \\ Aceito em 1 de Julho de 2009
}

\section{Resumo}

O presente estudo teve por objetivo investigar a associação de salinomicina e semduramicina, em diferentes doses, frente à infecçáo mista controlada de Eimeria acervulina, E. maxima e E. tenella em frangos de corte. Oitocentas aves foram divididas em 5 grupos (T1: ração não medicada; T2: 30 ppm de salinomicina e 12,5 ppm de semduramicina; T3: $30 \mathrm{ppm}$ de salinomicina e $15 \mathrm{ppm}$ de semduramicina; T4: $40 \mathrm{ppm}$ de salinomicina e 12,5 ppm de semduramicina e T5: 40 ppm de salinomicina e 15 ppm de semduramicina) e inoculadas aos 15 dias de idade com oocistos esporulados de E. acervulina, E. maxima e E. tenella, em inóculo misto, via ração. Parâmetros produtivos e escore de lesōes foram registrados. Todos os grupos tratados apresentaram estatisticamente melhores ganhos de peso cumulativo aos 21 dias de vida. Aos 35 dias de vida, somente o grupo T3 apresentou diferença significativa. A conversão alimentar cumulativa apresentou diferença estatística nos grupos T4 e T5. O tratamento T5 foi mais eficaz no controle de E. tenella. T3 e T5 obtiveram diferenças estatísticas no escore médio de lesão das três espécies. O uso de salinomicina, associada a semduramicina, em baixas doses demonstrou uma opção viável no controle da coccidiose neste experimento.

Palavras-chave: Eimeria, salinomicina, semduramicina, frango de corte, inóculo misto.

\begin{abstract}
This study aimed to investigate the association of salinomycin and semduramicin, in different doses, against controlled mixed infection of Eimeria acervulina, E. maxima and E. tenella in broiler chickens. Eight hundred birds were divided into 5 groups (T1: not medicated feed; T2: $30 \mathrm{ppm}$ of salinomycin and $12.5 \mathrm{ppm}$ of semduramicin; T3: $30 \mathrm{ppm}$ of salinomycin and $15 \mathrm{ppm}$ of semduramicin; T4: $40 \mathrm{ppm}$ of salinomycin and 12,5 ppm of semduramicin and T5: $40 \mathrm{ppm}$ of salinomycin and $15 \mathrm{ppm}$ of semduramicin) and inoculated at 15 days of age with sporulated oocysts of E. acervulina, E. maxima and E. tenella in a mixed suspension, through the feed. Performance data and lesion scores were recorded. All treated groups showed statistically better cumulative weight gain at 21 days old. At 35 days old only the T3 group showed significant difference. Cumulative feed conversion showed statistical difference in the groups T4 and T5. The treatment T5 was more effective in the coccidiosis control of E. tenella. T3 and T5 achieved statistical differences in the average lesion scores of the three analyzed species. The association of salinomycin and semduramicin used in lower doses than the usual, showed to be an option in the coccidiosis control in this experiment.
\end{abstract}

Keywords: Eimeria, salinomycin, semduramicin, broiler chicken, mixed infection.

\section{Introdução}

Entre as principais patologias da avicultura moderna, a coccidiose (ou eimeriose) é a mais importante causa de severas perdas para a indústria avícola (ASHRAF et al., 2002). A reduçâo da taxa de crescimento e o aumento da conversão alimentar, causados pela coccidiose, podem contribuir para um menor desempenho

\footnotetext{
*Autor para correspondência: Giankleber Strumielo Diniz

Universidade Estadual de Londrina - UEL,

Campus Universitário, CP 6001 CEP 86051-990, Londrina - PR, Brasil

e-mail: giankleber@hotmail.com
}

das aves comerciais. A produção intensiva moderna de frangos é altamente dependente de quimioprofilaxia para o controle da coccidiose (CHAPMAN, 1999), sendo o uso de anticoccídicos, especialmente os ionóforos em raçóes de frangos de corte, o método primário para a prevenção e controle dessa importante patologia (HOOGE et al., 1999).

A salinomicina foi lançada mundialmente em 1978 e, no Brasil, em 1981, tornando-se o ionóforo mais usado no controle 
da coccidiose aviária, por aliar eficácia e custo compatíveis. As doses indicadas para o controle da coccidiose são de 50 a $70 \mathrm{~g}$ por tonelada (ppm) nos alimentos de frangos de corte (FERREIRA; DELL'PORTO, 1999). A dose usual, no Brasil, situa-se entre 60 a 66 ppm (DINIZ, 2004). A eficácia da salinomicina no controle da coccidiose, a diminuição da presença de oocistos nas fezes, a recuperação das lesóes causadas pela patogenia, a melhoria no ganho de peso e conversão alimentar são amplamente demonstradas na literatura mundial (MOREHOUSE; BARRON, 1970; McDOUGALD et al., 1986; RADU et al., 1987; HARMS et al. 1989; McDOUGALD et al., 1990; CONWAY et al., 1993; CONWAY et al., 2001).

A semduramicina foi lançada no Brasil, em primeiro plano mundial, em 1995, antes dos Estados Unidos da América, Europa e Ásia. A dose indicada de semduramicina é de 20 a 30 g por tonelada (ppm) nos alimentos de frangos de corte (FERREIRA; DELL'PORTO, 1999; SCAN, 2002), mas, no Brasil, a dose usual é de 25 ppm (DINIZ, 2004). O controle da coccidiose aviária com o uso da semduramicina está descrito em várias publicaçóes, demonstrando o controle da enfermidade, diminuição dos oocistos, diminuição das lesóes da enfermidade, melhoria no ganho de peso e conversão alimentar (RICKETTS et al., 1992; McKENZIE et al., 1993; McDOUGALD et al., 1996; PESTI et al., 1999a; PESTI et al., 1999b; PESTI et al., 1999c; PESTI et al., 2002).

O presente estudo teve por objetivo investigar a associação de salinomicina e semduramicina, em diferentes doses, no tratamento das aves frente à infecção experimental mista de Eimeria acervulina, E. maxima e E. tenella em frangos de corte, como alternativa para o controle da coccidiose.

\section{Material e Métodos}

O presente trabalho foi realizado em galpão experimental constituído de 48 boxes, de 2,25 $\mathrm{m}^{2}$ cada, com capacidade para 20 aves por box $\left(8,8\right.$ aves por $\left.\mathrm{m}^{2}\right)$. Foram utilizados 800 pintinhos machos, de um dia de idade, linhagem comercial Ross, provenientes da Integração FRINAL (Garibaldi- RS), vacinados contra doença de Marek. Três fases de ração foram utilizadas, sem adição de matéria-prima de origem animal, divididas em: inicial (de 0 a 21 dias), crescimento (de 22 a 35 dias) e final (de 35 a 42 dias). A mistura de Salinomicina $\left(\right.$ Coxistac $^{\circledR}$ - Phibro Saúde Animal) e Semduramicina (Aviax ${ }^{\circledR}$ - Phibro Saúde Animal), conforme tratamentos abaixo, foi ofertada nas raçóes "inicial" e "crescimento", de zero a 35 dias de idade e a raçáo final livre de anticoccídicos para todos os tratamentos.

O alimento e água foram oferecidos ad libitum, em comedouros tubulares e bebedouros pendulares, respectivamente. Os níveis nutricionais utilizados foram baseados nos parâmetros empregados na indústria avícola nacional e descritos na Tabela 1 .

O teste realizado foi aprovado pelo comitê de ética da Universidade Federal de Santa Maria, RS, local de realização do estudo.

A preparação do inóculo foi realizada, no Laboratório de Parasitologia e Doenças Parasitárias do Departamento de Medicina Veterinária Preventiva da Universidade Estadual de Londrina, com
Tabela 1. Níveis nutricionais da ração oferecida aos frangos durante o experimento.

\begin{tabular}{lccc}
\hline \multicolumn{1}{c}{ Nutrientes } & Inicial & Crescimento & Final \\
\hline Proteína Bruta (\%) & 21 & 19,5 & 18 \\
En. Met (Kcal/kg) & 3.000 & 3.050 & 3.150 \\
Ca (\%) & 0,96 & 0,90 & 0,90 \\
P disp. (\%) & 0,48 & 0,45 & 0,40 \\
Met + Cis (\%) & 0,87 & 0,80 & 0,73 \\
Lisina (\%) & 1,16 & 1,05 & 0,94 \\
Treonina (\%) & 0,81 & 0,75 & 0,70 \\
Triptofano (\%) & 0,26 & 0,24 & 0,21 \\
Arginina (\%) & 1,36 & 1,24 & 1,13 \\
\hline
\end{tabular}

En. Met: energia metabolizável; Ca: cálcio; P disp: fósforo disponível; Met + Cist: metionina + cistina.

cepas isoladas de campo, conforme metodologia descrita por Long (1971) e, para cada ave, foi composto de $1,5 \times 10^{5}$ oocistos de E. acervulina, $5 \times 10^{4}$ oocistos de E. maxima e $1,5 \times 10^{4}$ oocistos de E. tenella, mantidos em dicromato de potássio $2,5 \%$. A composição do inóculo misto buscou a obtenção de escores médios de lesão de 1,5 a 2,5, com o objetivo de interferir na performance, sem que ocorra mortalidade elevada, segundo Conway et al. (1999).

A avaliação do escore médio de lesôes foi o resultado da média da soma dos escores de cada espécie, em cada ave, para demonstrar a intensidade de lesóes que o trato gastrintestinal apresenta em diferentes regióes do aparelho digestivo.

Aleatoriamente, as aves foram distribuídas em cinco tratamentos com oito repetições de 20 aves cada. Os tratamentos constituíram de: T1, grupo infectado e sem anticoccídico; T2, infectado e medicado com 30 ppm de salinomicina e 12,5 ppm de semduramicina; T3, infectado e medicado com $30 \mathrm{ppm}$ de salinomicina e $15 \mathrm{ppm}$ de semduramicina; T4, infectado e medicado com 40 ppm de salinomicina e 12,5 ppm de semduramicina; e T5, infectado e medicado com 40 ppm de salinomicina e 15 ppm de semduramicina. Não foram utilizadas aves não inoculadas e não medicadas, pois o trabalho objetivou o efeito da redução das lesóes em relação a aves infectadas e não à observação dos efeitos da coccidiose.

Foram separadas $40 \mathrm{~g}$ de ração por ave de cada tratamento e adicionado o inoculo. A raçáo com o inoculo, de cada tratamento, foi oferecida aos animais após quatro horas de jejum, em única dose, aos 15 dias de idade. Após a inoculação, retornou-se à administração de ração inicial de cada tratamento, sem o inoculo, até aos 21 dias de idade. Nesta idade, duas aves aleatórias de cada repetição foram eutanasiadas e necropsiadas para a avaliação de escores de lesão em três segmentos intestinais (duodeno, jejuno e ceco), segundo Johnson e Reid (1970). A seleção das aves e a avaliação foram realizadas em método duplo cego (apresentador das aves para a necropsia e responsável pela leitura dos escores de lesóes para coccidiose sem conhecimento do desenho experimental).

Os dados coletados, durante o experimento, foram: peso vivo, consumo de alimento e conversão alimentar ao zero, 21, 35 e 42 dias. Também foi registrada a mortalidade diária e os escores de lesóes aos 21 dias. 
A análise estatística foi realizada por meio do software Statgraphics, versão 5.0, sendo submetidos à análise de variância e, as médias, comparadas pelo teste de Tukey.

\section{Resultados e Discussão}

O consumo cumulativo de ração, de cada período, está demonstrado na Tabela 2, o qual náo foi afetado em nenhum dos períodos observados, diferente do observado por McDougald e Mequision (1980), em que houve um maior consumo de alimento em aves que receberam salinomicina (SAL), comparando-se ao grupo controle, porém não confirmado por Tipu et al., (2002). Quanto à semduramicina (SEM), níveis de 25 ppm não foram depressores no consumo de alimento (PESTI et al., 1999a; PESTI et al., 1999b; PESTI et al., 1999c). Desse modo, provavelmente, os resultados de uso dessa molécula, em doses menores, não afetaram o consumo da ração. A depressão do consumo de alimentos pela ação da associação de ionóforos, mesmo em pequena dose, foi demonstrada por Ebrahimnezhad e Pourreza (2005), em equaçôes de regressão de performance, quando comparadas ao uso de um ionóforo em sua dose normalmente ativa e determinada por testes de dose-eficácia. Esses resultados vêm ao encontro dos dados apresentados, quando comparados ao grupo controle deste trabalho.

A adição de SEM e SAL, associadas em todas as doses testadas, aumentou o ganho de peso corporal $(\mathrm{p}>0,05)$, no período de um a 21 dias de idade, quando comparadas ao controle infectado não medicado (Tabela 3). O grupo com as menores doses (T2) obteve o ganho de peso intermediário, diferente do grupo inoculado e não medicado com maiores dosagens (T5), porém náo foi diferente de T3 e T4. A maior dose de associação expressou o melhor ganho de peso (T5), provavelmente pelo melhor controle da coccidiose, bem como de infecçóes secundárias oriundas das lesôes gastrointestinais (ENGBERG et al., 2000; PEDROSO et al., 2006).

Aos 35 dias de idade, somente a adição de 30 ppm de SAL e 15 ppm de SEM manteve um maior ganho de peso corporal em relação à $\mathrm{T} 1$ ( $\mathrm{p}>0,05)$. O peso corporal, ao final do experimento, foi igual em todos os tratamentos. Aves inoculadas com oocistos de Eimeria e tratadas com salinomicina, em doses convencionais, apresentaram maior ganho de peso corporal, quando comparadas a aves inoculadas e não medicadas (CHANG et al., 1982; LOGAN, et al., 1993) o que não foi comprovado por outros autores (LEESON; SUMMERS, 1983; WELCH et al., 1988; ELWINGER et al., 1998). No uso isolado de SEM, um maior ganho de peso, comparado a aves inoculadas e não medicadas, também foi observado (PESTI et al., 2002; ISLAM et al., 2007). Diferenças estatísticas, relatadas em outros trabalhos científicos não foram demonstradas (PESTI et al., 1999a; PESTI et al., 1999c).

No experimento as aves foram mantidas até 42 dias de idade, o que possibilitou uma recuperação do ganho de peso. $\mathrm{Na}$ produção industrial, em que as fêmeas são abatidas com menor peso vivo e também aquelas para exportação ao Oriente Médio, onde o abate das aves ocorre aos 30 dias de idade, as diferenças na recuperação do ganho de peso podem representar um maior grau de importância.

Tabela 2. Consumo cumulativo de ração pelos frangos ao final de cada período experimental.

\begin{tabular}{ccccccc}
\hline \multicolumn{7}{c}{ Período (dias) } \\
\hline Tratamento* $^{c} \mathbf{1 - 2 1}$ & CV \% & $\mathbf{1 - 3 5}$ & $\mathbf{C V} \%$ & $\mathbf{1}-\mathbf{4 2}$ & $\mathbf{C V} \%$ \\
\hline 1 & $0,962 \pm 0,039$ & 4,068 & $2,787 \pm 0,082$ & 2,929 & $3,937 \pm 0,125$ & 3,175 \\
2 & $0,993 \pm 0,033$ & 3,300 & $2,768 \pm 0,070$ & 2,524 & $3,950 \pm 0,077$ & 1,957 \\
3 & $1,003 \pm 0,038$ & 3,779 & $2,884 \pm 0,084$ & 2,923 & $4,053 \pm 0,096$ & 2,363 \\
4 & $0,964 \pm 0,014$ & 1,461 & $2,775 \pm 0,067$ & 2,406 & $3,908 \pm 0,083$ & 2,112 \\
5 & $0,980 \pm 0,030$ & 3,101 & $2,832 \pm 0,076$ & 2,693 & $3,918 \pm 0,134$ & 3,431 \\
CV \% & 3,646 & & 3,066 & & 3,117 \\
\hline
\end{tabular}

*T1: inoculado e não medicado; T2: $30 \mathrm{ppm}$ de salinomicina e 12,5 ppm de semduramicina; T3: $30 \mathrm{ppm}$ de salinomicina e 15 ppm de semduramicina; T4: 40 ppm de salinomicina e 12,5 ppm de semduramicina; T5: $40 \mathrm{ppm}$ de salinomicina e $15 \mathrm{ppm}$ de semduramicina. CV: coeficiente de variação. Não houve diferenças estatísticas $(\mathrm{P}<0,05)$.

Tabela 3. Ganho cumulativo de peso corporal dos frangos ao final de cada período experimental e percentual de mortalidade ao final do experimento.

\begin{tabular}{|c|c|c|c|c|c|c|c|c|}
\hline \multicolumn{9}{|c|}{ Período (dias) } \\
\hline Tratamento* & $1-21$ & $\mathrm{CV} \%$ & $1-35$ & $\mathrm{CV} \%$ & $1-42$ & CV \% & $\%$ Mortalidade aos 42 dias & CV\% \\
\hline 1 & $0,603 \pm 0,015 c$ & 2,510 & $1,576 \pm 0,035 \mathrm{~b}$ & 2,241 & $2,085 \pm 0,042$ & 2,020 & $3,473 \pm 5,089$ & 146,562 \\
\hline 2 & $0,635 \pm 0,015 b$ & 2,418 & $1,591 \pm 0,029 \mathrm{ab}$ & 1,798 & $2,101 \pm 0,024$ & 1,136 & $2,780 \pm 2,972$ & 106,904 \\
\hline 3 & $0,656 \pm 0,021 \mathrm{ab}$ & 3,204 & $1,662 \pm 0,064 \mathrm{a}$ & 3,870 & $2,167 \pm 0,063$ & 2,888 & $2,779 \pm 4,200$ & 151,152 \\
\hline 4 & $0,642 \pm 0,018 \mathrm{ab}$ & 2,800 & $1,611 \pm 0,064 \mathrm{ab}$ & 3,991 & $2,120 \pm 0,072$ & 3,410 & $2,085 \pm 2,878$ & 138,013 \\
\hline 5 & $0,665 \pm 0,015 a$ & 2,298 & $1,629 \pm 0,052 \mathrm{ab}$ & 3,201 & $2,094 \pm 0,067$ & 3,204 & $2,085 \pm 2,878$ & 138,013 \\
\hline $\mathrm{CV} \%$ & 4,035 & & 3,517 & & 2,955 & & 123,473 & \\
\hline
\end{tabular}

*T1: inoculado e não medicado; T2: 30 ppm de salinomicina e 12,5 ppm de semduramicina; T3: 30 ppm de salinomicina e 15 ppm de semduramicina; T4: 40 ppm de salinomicina e 12,5 ppm de semduramicina; T5: 40 ppm de salinomicina e 15 ppm de semduramicina. CV: coeficiente de variação. Médias nas colunas seguidas por letras diferentes são estatisticamente significativas $(\mathrm{P}<0,05)$. 
A associação de dois ionóforos, em doses menores que as recomendadas de cada um deles, parecem promover maior ganho de peso que o uso das drogas em doses completas, dados demonstrados por Ebrahimnezhad e Pourreza (2005), na associação de SAL e lasalocida. Em virtude do complexo modo de ação dos ionóforos, a resistência a esses compostos não ocorre rapidamente (KAWAZOE, 2009), porque está diretamente ligada às características farmacológicas, tempo de exposição, prevenção e rotatividade dos fármacos utilizados (FERREIRA; DELL'PORTO, 1999). O uso de anticoccídicos ionóforos, em doses menores que as recomendadas, é uma importante prática adotada em matrizes ou reprodutoras pesadas e poedeiras comerciais, como auxiliar no desenvolvimento de imunidade contra a coccidiose (KAWAZOE, 2009).

$\mathrm{Na}$ Tabela 3, evidencia-se que a mortalidade, ao final do período, não apresentou diferenças entre os diversos tratamentos.

Os tratamentos com níveis de 40 ppm de SAL (T4 e T5) demonstraram uma melhor conversão alimentar, aos 21 dias de idade ( $p>0,05$ ), comparados ao controle inoculado e não medicado, sendo que o tratamento de maiores doses, com 40 ppm de SAL e 15 ppm de SEM (T5), obteve também diferença estatística em relação ao tratamento de menor dose de associação, 30 ppm de SAL e 12,5 ppm de SEM (T2).

Considerando-se a conversão alimentar cumulativa, nos períodos de 35 e de 42 dias de idade, os tratamentos não revelaram diferenças. Esses resultados sáo apresentados na Tabela 4. No entanto a melhoria na conversão alimentar com o uso isolado de SAL foi constatada por Chang et al. (1982) e Conway et al. (1995), porém está em desacordo com os dados encontrados por outros pesquisadores (KESHAVARZ; McDOUGALD, 1982; HARMS et al., 1988;
EBRAHIMNEZHAD; POURREZA, 2005). Quanto à SEM, utilizada isoladamente, os dados da literatura também são contrastantes, nos quais uma melhor conversão alimentar em frangos de corte é observada em alguns experimentos (CONWAY et al., 1995; McDOUGALD et al., 1996; PESTI et al., 2002) e não comprovada em outros (PESTI et al., 1999a; PESTI et al., 1999b; PESTI et al., 1999c; ISLAM et al., 2007).

Os escores de lesáo para E. acervulina e E. maxima não apresentaram diferenças estatísticas entre os tratamentos, entretanto as aves do grupo T5, com 40 ppm de SAL e 15 ppm de SEM, demonstraram menor escore para E. tenella $(\mathrm{p}>0,05)$ que as aves dos outros tratamentos (Tabela 5). Esse resultado pode ser atribuído a maior eficácia da SEM no controle da E. tenella (DINIZ, 2008). O efeito positivo da SEM, isoladamente no controle dos escores de lesão de E. tenella, já foi demonstrado anteriormente (McKENZIE et al., 1993; McDOUGALD et al., 1996; SCAN, 2002; KOINARSKI, 2003), bem como o efeito da SAL na diminuição do escore de lesôes em aves inoculadas e não medicadas (CHANG et al., 1982; JO; JANG, 1988; CONWAY et al., 1995).

A utilização de inóculo misto busca simular condições de campo e possibilita praticidade na inoculação, podendo-se, nessa modalidade de inoculação, avaliar o escore de lesóes de cada espécie, escore médio de lesóes e contagem de oocistos (HUME et al., 2006; OVIEDO-RONDÓN et al., 2006a; OVIEDO-RONDÓN et al., 2006b; PARKER et al., 2007). A avaliação do escore médio de lesôes demonstrou uma diferença significativa para os tratamentos com níveis de 15 ppm de SEM (T3 e T5), os quais obtiveram menores escores médios de

Tabela 4. Conversão alimentar cumulativa dos frangos ao final de cada período experimental.

\begin{tabular}{|c|c|c|c|c|c|c|}
\hline \multirow[b]{2}{*}{ Tratamento* } & \multicolumn{6}{|c|}{ Período (dias) } \\
\hline & $1-21$ & CV \% & $1-35$ & $\mathrm{CV} \%$ & $1-42$ & $\mathrm{CV} \%$ \\
\hline 1 & $1,433 \pm 0,086 \mathrm{c}$ & 5,971 & $1,769 \pm 0,060$ & 3,386 & $1,888 \pm 0,050$ & 2,626 \\
\hline 2 & $1,392 \pm 0,027 \mathrm{bc}$ & 1,959 & $1,740 \pm 0,029$ & 1,670 & $1,880 \pm 0,023$ & 1,222 \\
\hline 3 & $1,372 \pm 0,047 \mathrm{abc}$ & 3,402 & $1,736 \pm 0,031$ & 1,791 & $1,871 \pm 0,025$ & 1,358 \\
\hline 4 & $1,348 \pm 0,024 \mathrm{ab}$ & 1,796 & $1,723 \pm 0,031$ & 1,823 & $1,845 \pm 0,031$ & 1,669 \\
\hline 5 & $1,325 \pm 0,013 \mathrm{a}$ & 0,992 & $1,739 \pm 0,037$ & 2,142 & $1,871 \pm 0,036$ & 1,910 \\
\hline $\mathrm{CV} \%$ & 3,950 & & 2,300 & & 1,925 & \\
\hline
\end{tabular}

*T1: inoculado e não medicado; T2: 30 ppm de salinomicina e 12,5 ppm de semduramicina; T3: 30 ppm de salinomicina e 15 ppm de semduramicina; T4: 40 ppm de salinomicina e 12,5 ppm de semduramicina; T5: 40 ppm de salinomicina e 15 ppm de semduramicina. CV: coeficiente de variação. Médias nas colunas seguidas por letras diferentes são estatisticamente significativas $(\mathrm{P}<0,05)$.

Tabela 5. Escore de lesóes aos 21 dias para Eimeria acervulina, E. maxima e E. tenella.

\begin{tabular}{ccccccccc}
\hline Tratamento* $^{\text {E. acervulina }}$ & CV \% & E. maxima & CV \% & E. tenella & CV \% & Escore médio de lesão** & CV\% \\
\hline 1 & $1,688 \pm 0,873$ & 51,746 & $1,563 \pm 0,964$ & 61,692 & $1,188 \pm 0,750 \mathrm{~b}$ & 63,158 & $1,478 \pm 0,595 \mathrm{~b}$ & 40,27 \\
2 & $1,500 \pm 1,265$ & 84,327 & $1,313 \pm 0,946$ & 72,113 & $0,438 \pm 0,629 \mathrm{ab}$ & 143,806 & $1,083 \pm 0,464 \mathrm{ab}$ & 42,83 \\
3 & $1,125 \pm 1,204$ & 107,036 & $0,875 \pm 1,025$ & 117,108 & $0,688 \pm 1,014 \mathrm{ab}$ & 147,561 & $0,895 \pm 0,594 \mathrm{a}$ & 66,36 \\
4 & $1,813 \pm 1,167$ & 64,401 & $1,000 \pm 0,894$ & 89,443 & $1,125 \pm 0,806 \mathrm{~b}$ & 71,665 & $1,312 \pm 0,507 \mathrm{ab}$ & 38,68 \\
5 & $1,625 \pm 0,957$ & 58,919 & $0,750 \pm 0,775$ & 103,280 & $0,188 \pm 0,403 \mathrm{a}$ & 214,994 & $0,854 \pm 0,438 \mathrm{a}$ & 51,27 \\
CV \% & 68,791 & & 87,485 & & 113,897 & 113,897 & \\
\hline
\end{tabular}

*T1: inoculado e nâo medicado; T2: 30 ppm de salinomicina e 12,5 ppm de semduramicina; T3: 30 ppm de salinomicina e 15 ppm de semduramicina; T4: 40 ppm de salinomicina e 12,5 ppm de semduramicina; T5: 40 ppm de salinomicina e 15 ppm de semduramicina. CV: coeficiente de variação. ** Escore médio de lesốes - média de escore de lesão das três espécies em cada ave para cálculo da média do grupo. Médias nas colunas, seguidas por letras diferentes, são estatisticamente significativas $(\mathrm{P}<0,05)$. 
lesôes quando comparados ao grupo controle. Esses resultados devem-se, provavelmente, aos baixos escores de E. maxima e E. tenella produzidos por esses tratamentos, uma vez que a SEM é mais eficaz no controle dessas espécies quando comparada à SAL (DINIZ, 2008).

\section{Conclusóes}

O uso de 30 ou $40 \mathrm{ppm}$ de salinomicina, associada a $15 \mathrm{ppm}$ de semduramicina, diminuiu o escore médio de lesões em presença de uma infecção mista por Eimeria. Novos estudos com diferentes combinaçôes de dosagens e inoculaçôes de cada espécie de Eimeria, isoladamente, poderão ajudar a entender melhor essas associaçôes.

\section{Referências}

ASHRAF, M. A. et al. Comparative efficacy of different feed additive anticoccidials in broilers. International Journal of Poultry Science, v. 1, n. 6, p. 185-187, 2002.

CHANG, K. S.; LIAO, C. F; YANG, C. P. Efficacy of salinomycin for controlling coccidiosis in broilers in subtropical Taiwan. Memoires of the College of Agriculture National Taiwan Universit, v. 22, p. 22-30, 1982.

CHAPMAN, H. D. Anticoccidial drugs and their effects upon the development of immunity to Eimeria infections in poultry. Avian Pathology, v. 28, n. 6, p. 521-535, 1999.

CONWAY, D. P.; DAYTON, A. D.; MCKENZIE, M. E. Comparative testing of anticoccidials in broiler chickens: the role of coccidial lesion scores. Poultry Science, v. 78, n. 4, p. 529-535, 1999.

CONWAY, D. P. et al. Efficacy of semduramicin and salinomycin against Eimeria maxima in a laboratory test using two levels of oocyst inocula. Poultry Science, v. 74, n. 12, p. 1942-1947, 1995.

CONWAY, D. P. et al. Efficacy of Semduramicin and Salinomycin against different stages of Eimeria tenella and Eimeria acervulina in the chicken. Veterinary Parasitology, v. 45, n. 3-4, p. 215-229, 1993.

CONWAY, D. P. et al. Efficacy of diclazuril in comparison with chemical and ionophorous anticoccidials against Eimeria spp. in broiler chickens in floor pens. Poultry Science, v. 80, n. 4, p. 426-430, 2001.

DINIZ, G. S. Controle da coccidiose: atualização técnica. Artigos Zoonews, 2004. Disponível em: < http://www.zoonews.com.br/noticiax. php?idnoticia=38824> Acesso em: 17 Janeiro 2007.

DINIZ, G. S. Eficácia dos ionóforos no controle da coccidiose. Artigos Zoonews, 2008. Disponível em: <http://www.zoonews.com.br/noticiax. php?idnoticia=130687 > Acesso em: 08 Janeiro 2008.

EBRAHIMNEZHAD, Y.; POURREZA, J. Effects of ionophorous anticoccidial drugs, salinomycin and lasalocid, on the performance of broiler chicks and the relationship of these drugs to supplementary methionine. International journal of Poultry Science, v. 4, n. 11, p. 911-916, 2005.

ELWINGER, K. et al. Effect of antibiotic growth promoters and anticoccidials on growth of Clostridium perfringens in the caeca and on the performance of broiler chickens. Acta Veterinaria Scandinavica, v. 39, n. 4, p. 433-441, 1998.
ENGBERG, R. M. et al. Effect of zinc bacitracin and salinomycin on intestinal microflora and performance of broilers. Poultry Science, v. 79, n. 9, p. 1311-1319, 2000.

FERREIRA, A. J.; DELL'PORTO, A. Agentes antiprotozoários. In: SPINOSA, H. S.; GORNIAK, S. L.; BERNARDI, M. M. Farmacologia Aplicada à Medicina Veterinária. 2 ed. Rio de Janeiro: Editora Guanabara Koogan S.A., 1999. p. 465-475.

HARMS, R. H.; RUIZ, N.; BURESH, R. E. Influence of monensin and salinomycin on the performance of broiler chicks. Poultry Science, v. 68, n. 1, p. $86-88,1989$.

HOOGE, D. M.; CUMMINGS, K. R.; McNAUGHTON, J. L. Evaluation of sodium bicarbonate, chloride, or sulfate with a coccidiostat in corn-soy or corn-soy-meat diets for broiler chickens. Poultry Science, v. 78 , n. 9, p. $1300-1306,1999$.

HUME, M. E.; CLEMENTE-HERNANDÉZ， S.; OVIEDO-RONDÓN, E. O. Effects of feed additives and mixed Eimeria species infection on intestinal microbial ecology of broilers. Poultry Science, v. 85, n. 12, p. 2106-2111, 2006.

ISLAM, K. M. S. et al. Yield carcass and sensory characteristics of broiler meat after withdrawal of anticoccidial Semduramicin from feed. Pakistan Journal of Nutrition, v. 6, n. 3, p. 276-282, 2007.

JO, Y. W.; JANG, D. H. Studies on the anticoccidial efficacy of polyether ionophorous antibiotics, Salinomycin sodium, monensin sodium and maduramycin ammonium by the anticoccidial index in chickens. Veterinary Bulletin, v. 58, p. 1934, 1988.

JOHNSON, J.; REID, W. M. Anticoccidial Drugs: lesion scoring techniques in battery and floor-pen experiments with chickens. Experimental Parasitology, v. 28, n. 1, p. 30-36, 1970.

KAWAZOE, U. Coccidiose. In: BERCHIERI Jr., A. et al. Doença das Aves. 2 ed. Campinas: Editora Facta, 2009. p. 837-858.

KESHAVARZ, K.; McDOUGALD, L. R. Anticoccidial drugs: growth and performance depressing effects in young chickens. Poultry Science, v. 61, n. 4, p. 699-705, 1982.

KOINARSKI, V. A comparative study on anticoccidial efficiency of semduramicin in broiler chickens infected with Eimeria tenella. Bulgarian. Journal of Veterinary Medicine, v. 6, n. 2, p. 111-117, 2003.

LEESON, S.; SUMMERS, J. D. Growth and carcass characteristics of broilers fed salinomycin in diets containing graded levels of methionine and energy. Canadian Journal of Animal Science, v. 63, p. 409-419, 1983.

LOGAN, N. B. et al. Anticoccidial efficacy of semduramicin. 2. Evaluation against field isolates including comparisons with salinomycin, maduramicin and monensin in battery tests. Poultry Science, v. 72, n. 11, p. 2058-2063, 1993.

LONG, P. L. Maintenance of intestinal protozoa in vivo with particular reference to Eimeria and Histomonas. In: TAYLOR, A. E. R.; MULLER, $R$. Isolation and maintenance of parasites in vivo. Oxford: Blackwell Scientific Publication, 1971. p. 65-75.

McDOUGALD, L. R.; FULLER, A. L.; SOLIS, J. Drug sensitivity of 99 isolates of coccidia from broiler farms. Avian Disease, v. 30, n. 4, p. 690-694, 1986.

McDOUGALD, L. R.; MATHIS, G. F.; CONWAY, D. P. Effect of semduramicin, salinomycin and monensin on performance, shank pigmentation, and coccidial lesions in broiler chickens in floor pens. Avian Disease, v. 40, n. 1, p. 68-71, 1996. 
McDOUGALD, L. R.; MEQUISION, T. E. Compensatory growth in broilers after withdrawal of ionophorous anticoccidial drugs. Poultry Science, v. 59, n. 5, p. 1001-1005, 1980.

McDOUGALD, L. R. et al. Anticoccidial efficacy of diclazuril in broilers under simulated natural conditions in floor pens. Avian Disease, v. 34, n. 4, p. 905-910, 1990.

McKENZIE, M. E. et al. Anticoccidial efficacy of semduramicin: 1. evaluation against field isolates by dose titration in battery tests. Poultry Science, v. 72, n. 11, p. 2052-2057, 1993.

MOREHOUSE, N. F.; BARRON, R. R. Coccidiosis: evaluation of coccidiostats by mortality, weight gains, and fecal scores. Experimental Parasitology, v. 28, n. 1, p. 25-29, 1970.

OVIEDO-RONDÓN, E. O. et al. Essential oils on mixed coccidia vaccination and infection in broilers. International Journal of Poultry Science, v. 5, n. 8, p. 723-730, 2006a.

OVIEDO-RONDÓN, E. O. et al. Intestinal microbial ecology of broilers vaccinated and challenged with mixed Eimeria species, and supplemented with essential oil blends. Poultry Science, v. 85, n. 5, p. $854-860,2006 \mathrm{~b}$.

PARKER, J. et al. Enzymes as feed additive to aid in responses against eimeria species in coccidia-vaccinated broilers fed corn-soybean meal diets with different protein levels. Poultry Science, v. 86, n. 4, p. 643-653, 2007.

PEDROSO, A. A. et al. Intestinal bacterial community and growth performance of chickens fed diets containing antibiotics. Poultry Science, v. 85, n. 4, p. 747-752, 2006.

PESTI, G. M. et al. Studies on Semduramicin and nutritional responses: 1. level and source of protein. Poultry Science, v. 78, n. 1, p. 102-106, 1999a.
PESTI, G. M. et al. Studies on Semduramicin and nutritional responses: 2. methionine levels. Poultry Science, v. 78, n. 8, p. 1170-1176, $1999 \mathrm{~b}$.

PESTI, G. M. et al. Studies on Semduramicin and nutritional responses: 3. electrolyte balance. Poultry Science, v. 78, n. 11, p. 1552-1560, 1999c.

PESTI, G. M. et al. The influence of withdrawal time on the performance of broiler chickens fed semduramicin. Poultry Science, v. 81, n. 7 , p. 939-944, 2002.

RADU, J. et al. Feed and water consumption and performance of male and female broilers fed salinomycin and maduramicin followed by a withdrawal ration. Poultry Science, v. 66, n. 11, p. 1878-1881, 1987.

RICKETTS, A. P.; OLSON, J. A.; RICE, J. R. In vivo expression of in vitro anticoccidial activity. Antimicrobial Agents and Chemotherapy, v. 36, n. 10, p. 2338-2341, 1992.

SCAN, 2002. Report of the Scientific Committee on Animal Nutrition on the use of semduramicin sodium in feedingstuffs for chickens for fattening. Scientific Committee on Animal Nutrition, p. 18, 2002.

TIPU, M. A.; PASHA, T. N.; ALI, Z. Comparative efficacy of salinomycin sodium and neem fruit (Azadirachta indica) as feed additive anticoccidials in broilers. International Journal of Poultry Science, v. 1, n. 4, p. 91-93, 2002.

WELCH, C. C.; PARSONS, C. M.; BAKER, D. H. Further investigation of the dietary protein level-monensin interrelationship in broiler chicks: Influence of dietary protein source and type of anticoccidial drug. Poultry Science, v. 67, n. 4, p. 652-659, 1988. 